\title{
Multiferroic interfaces in bismuth ferrite composite fibers grown by laser floating zone technique
}

\author{
F.G. Figueiras ${ }^{\text {a,b,* }}$, D. Dutta ${ }^{\text {a }}$, N.M. Ferreira ${ }^{\text {a }}$, F.M. Costa ${ }^{\text {a }}$, M.P.F. Graça ${ }^{\text {a }}$, M.A. Valente ${ }^{\text {a }}$ \\ a Physics Department \& I3N, Aveiro University, 3810-193 Aveiro, Portugal \\ b Physics Department \& CICECO, Aveiro University, 3810-193 Aveiro, Portugal
}

\section{A R T I C L E I N F O}

\section{Article history:}

Received 3 February 2015

Received in revised form 9 November 2015

Accepted 11 November 2015

Available online 12 November 2015

\section{Keywords:}

Multiferroic

Interfaces

$\mathrm{BiFeO}_{3}$

Composite

Laser floating zone

\begin{abstract}
A B S T R A C T
In this work we explore the formation of enhanced multiferroic interfaces in bismuth ferrite crystalline fibers grown by laser floating zone technique. An underlying mechanism of self-segregation during the fibers growth process enables to establish a textured microstructure of a dominant $\mathrm{BiFeO}_{3}$ phase bordered by the presence of $\mathrm{Bi}_{25} \mathrm{FeO}_{40}$ secondary phase. The crystallites $\boldsymbol{c}$ axis of the $\mathrm{BiFeO}_{3}$ phase shows a preferential orientation along the longitudinal axis of the fibers, together with grain boundaries that also present a significant alignment with the same direction. These features induce a systematic disturbance of the antiferromagnetic structure of the $\mathrm{BiFeO}_{3}$ phase at the interfaces with the $\mathrm{Bi}_{25} \mathrm{FeO}_{40}$ diamagnetic phase. The structural anisotropy confirmed by High Resolution X-ray diffraction and scanning electron microscopy images is also manifested in the magnetic properties of the fibers, which reveal an enhanced susceptibility response in comparison to the conventional $\mathrm{BiFeO}_{3}$ phase diagram.
\end{abstract}

(c) 2015 Elsevier Ltd. All rights reserved.

\section{Introduction}

A considerable focus have been given to the metal oxide materials as they manifest a broad range of structural and exciting physical properties [1,2]. Only small subgroups of all magnetically and electrically polarizable materials are either ferromagnetic or ferroelectric and fewer still simultaneously exhibit both order parameters [3]. Among many of the promising functional responses exhibited by a few of these materials is the existence of two or more "ferroic" order parameters simultaneously (ferroelectricity, anti/ferromagnetism, ferroelasticity), whereas the degree of coupling between the magnetic and polarization properties is classified as magnetoelectric effect [4]. Such is the case of single-phase multiferroic $\mathrm{BiFeO}_{3}$ among other like rare earth manganites (e.g. $\mathrm{TbMnO}_{3}, \mathrm{HoMnO}_{3}$ ) or $\mathrm{BaNiF}_{4}$ or even chalcogenides like $\mathrm{ZnCr}_{2} \mathrm{Se}_{4}$. Most of these interesting materials are found to be in a group of pseudo-perovskite structure, characterized by a general chemical formula $\mathrm{ABO}_{3}$ (e.g., $\mathrm{CaTiO}_{3}, \mathrm{SrRuO}_{3}, \mathrm{BiFeO}_{3}$ ) comprising cornersharing six oxygen octahedral with a central B-cation and a A-cation that can coordinate with up to twelve oxygen ions. The particular case of $\mathrm{BiFeO}_{3}$ (BFO) attracted much attention, as it is essentially the only known multiferroic that simultaneously possesses both magnetic and ferroelectric order at and above room temperature [5].

$\mathrm{BiFeO}_{3}$ has a rhombohedral unit cell characterized by two distorted perovskite blocks connected along their body diagonal [ 1111 ], where the two oxygen octahedra of the two cells are rotated clockwise and

\footnotetext{
* Corresponding author.

E-mail address: ffigueiras@ua.pt (F.G. Figueiras).
}

counterclockwise around the $\left[\begin{array}{lll}1 & 1 & 1\end{array}\right]$ by $\pm 13.8(3)^{\circ}$ and the Fe-ion is shifted by $13.5 \mathrm{pm}$ along the same axis [6]. $\mathrm{BiFeO}_{3}$ is a robust antiferromagnetic-ferroelectric with a cycloid spin structure having a period of $62 \mathrm{~nm}$ [7]. The symmetry also permits a small canting of the moments in the structure resulting in a weak canted ferromagnetic moment of the Dzyaloshinskii-Moriya type [8,9]. Spurred on by a 2003 paper focusing on the growth and properties of $\mathrm{BiFeO}_{3}$ thin films [10], dramatic advances in the study and understanding of this material have occurred. Much work is available on the magnetic, magnetoelectric and magneto transport [11] properties of the $\mathrm{BiFeO}_{3}$ films as a function of the growth parameters [12] and there exist different thermodynamic (e.g. Landautype) models $[13,14]$ to examine the extent of contribution of domain walls in the enhancement of magnetization in these films. He et al. [11] have also demonstrated that in magneto transport certain types of domain walls (i.e., $109^{\circ}$ walls) can exhibit strong temperature- and magnetic field-dependent magneto resistance (as large as 60\%) which is thought to be the result of local symmetry breaking at domain walls and the formation of magnetic moments. Not much detailed work on the properties of the bulk BFO ceramics is available because of the difficulty in reducing the secondary phases obtained, during the preparation of crystalline $\mathrm{BiFeO}_{3}$ bulks by several methods such as sol-gel [15], solid state reaction [16], simple precipitation [17], rapid liquid phase sintering [18], chemical solution deposition [19] and high-energy ball milling [20].

The laser floating zone (LFZ) technique is a well-known method to grow large, clean and homogeneous single crystals, particularly considering that a high temperature gradient ahead to the solidification interface can lead to the formation of single crystals with high quality of 
crystallinity [21,22]; while at a higher growth rate it also enables to synthesize textured crystal fibers with enhanced anisotropic physical properties $[23,24]$. The main parameters that have large influence on the quality of the crystal grown by this technique are the temperature gradient, the growth rate and atmosphere. The LFZ technique allows producing highly textured materials with superior properties when compared to their sintered forms, which produce drawbacks such as disordered grain boundaries and high anisotropy in the charge transport [25-27]. In this study, we used the LFZ technique to produce fibers from initial nominal composition $\mathrm{Bi}_{1.2} \mathrm{Fe}_{0.8} \mathrm{O}_{3}$, in an attempt to obtain the stoichiometric phase of $\mathrm{BiFeO}_{3}$. The stoichiometry of precursor materials is chosen based on the study by Pradhan et al. [28] in order to compensate the effect of vaporization loss of Bismuth during the high temperature melting in the LFZ. Our study focuses on the effect of the fibers growth rate on phase development, texturing and magnetic properties.

\section{Experimental procedures}

Appropriate amount of $\mathrm{Bi}_{2} \mathrm{O}_{3}$ and $\mathrm{Fe}_{2} \mathrm{O}_{3}$ powders from Aldrich (purity $>99 \%$ ) to obtain the $\mathrm{Bi}_{1.2} \mathrm{Fe}_{0.8} \mathrm{O}_{3}$ composition, were mixed with ethanol and ball milled for $40 \mathrm{~min}$ at $250 \mathrm{rpm}$. The obtained homogenous mixture was dried at $100{ }^{\circ} \mathrm{C}$, then added to a $2 \%$ polyvinyl alcohol (PVA) binder enabling the precursor to be extruded into rods of $2 \mathrm{~mm}$ diameter [29]. These rods are used as feed and seed in the LFZ growth process in normal room atmosphere. The LFZ system used is equipped with a continuous $\mathrm{CO}_{2}$ Spectron SLC laser $(\boldsymbol{\lambda}=10.6 \mu \mathrm{m} ; 200 \mathrm{~W})$ suitable to grow dense fibers. In order to provide better homogeneity of the target fibers, the seed and feed rod precursors rotated respectively at $\omega_{s}=0.166 \pi$ and $\omega_{f}=0.5 \pi \mathrm{s}^{-1}$ (5 and $15 \mathrm{rpm}$ ) in opposite directions,. Three different growth rates were analyzed in detail: 25,50 and $100 \mathrm{~mm} / \mathrm{h}$. X-ray diffraction (XRD) was performed using a X'Pert MPD Philips diffractometer ( $\mathrm{Cu} \mathrm{K} \alpha$ radiation, $\lambda=0.154056 \mathrm{~nm}$ ) at $40 \mathrm{kV}$ and $30 \mathrm{~mA}$, based in a Bragg-Brentano para-focusing optics configuration. Rietveld refinement analysis [30] of diffractograms enables to estimate phase's proportion and crystallographic structure. Surface morphology of the fibers was observed using a Hitachi S41001 scanning electron microscopy (SEM), and phase's composition analyzed using energy dispersion spectroscopy (EDS) mode $(25 \mathrm{kV}$, $10 \mathrm{~mA}$ ). The magnetic susceptibility measurements were performed using a Cryogenics vibrating sample magnetometer (VSM). The magnetic properties as a function of temperature were acquired in field cooling (FC) and zero field cooling (ZFC) modes, applying a magnetic field of 0.5 Tesla, and magnetization versus magnetic field at constant temperatures of 5 and $300 \mathrm{~K}$. The magnetic measurements were made in two different geometries, by placing the samples in a parallel and perpendicular position relative to the direction of the applied magnetic field [31].

\section{Results and analysis}

Fig. 1 shows the X-ray powder diffraction pattern obtained for fiber grown at $25 \mathrm{~mm} / \mathrm{h}$ by LFZ. According to the respective Rietveld analysis the majority phase matches the $\mathrm{BiFeO}_{3}$ pseudo-perovskite, in agreement to the results obtained by Pradhan [28], indexed to the SG R3c $h$ (161) [32]; in parallel, a considerable amount ( 40\%) of secondary phase can be indexed to the $\mathrm{Bi}_{25} \mathrm{FeO}_{40}$ I23 (197) cubic phase [33]. Fig. 1 also compares the XRD diffractograms taking in consideration the fiber cross sections. For the measurement performed in a transversal section, planes like ( $\left.\begin{array}{lll}0 & 0 & 6\end{array}\right),\left(\begin{array}{lll}0 & 1 & 8\end{array}\right)$ and $\left(\begin{array}{lll}1 & 0 & 10\end{array}\right)$ of $\mathrm{BiFeO}_{3}$ phase are highly enhanced when compared to the measurement obtained from the longitudinal cut geometry, which exhibits as main peaks planes ( 1 $10)$ and (2 111 ) indicative of a preferred orientation of $\boldsymbol{c}$ axis parallel to the fiber growth direction $(\boldsymbol{z})$. In its turn, $\mathrm{Bi}_{25} \mathrm{FeO}_{40}$ phase profiles are much similar in both diffractograms as inherent from a cubic symmetry.

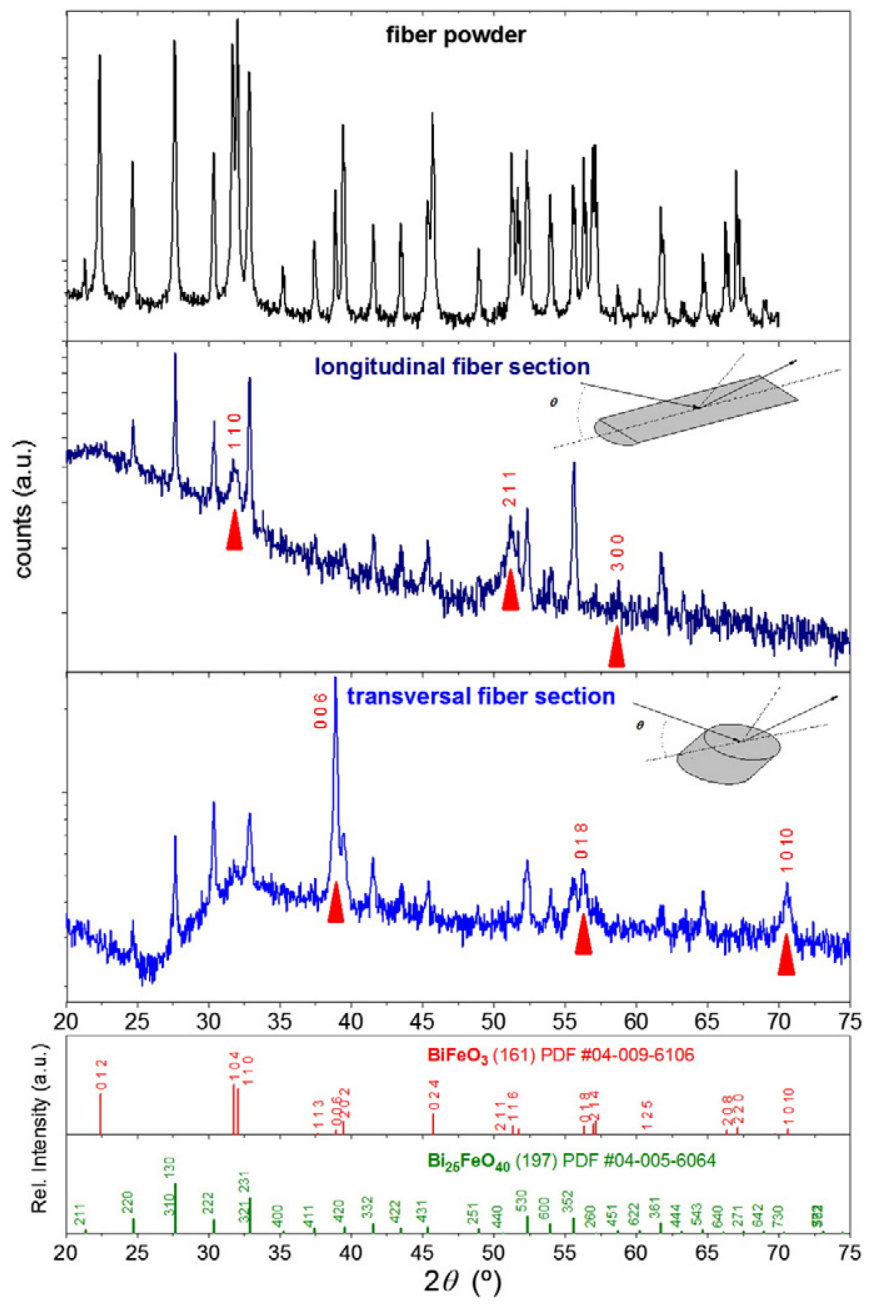

Fig. 1. Comparison of X-ray diffractograms obtained from the fiber grown by LFZ at $25 \mathrm{~mm} / \mathrm{h}$, for the powder, longitudinal and transversal sections, respectively indexed to $\mathrm{BiFeO}_{3}$ and $\mathrm{Bi}_{25} \mathrm{FeO}_{40}$ phases.

Table 1 compares the structural results calculated from the diffractograms obtained from the 25,50 and $100 \mathrm{~mm} / \mathrm{h}$ fibers growth speed. A perceptible increase (up to $\sim 5 \%$ ) of the secondary $\mathrm{Bi}_{25} \mathrm{FeO}_{40}$ phase can be associated with the fibers processing rising speed.

Further confirmation and scrutiny of the structure can be obtained from pole figures; the preferable orientation of the $\left(\begin{array}{ll}0 & 0\end{array}\right)$ planes of crystallites is visible from padding of peaks at $2 \boldsymbol{\theta}=39.0^{\circ}$ within $\Delta \psi<30^{\circ}$ dispersion as shown in Fig. 2a. In Fig. 2b the reflections of (1 10 ) planes at $2 \boldsymbol{\theta}=32.0^{\circ}$ display a consistent distribution $\Delta \boldsymbol{\psi}<30^{\circ}$ along the fiber main direction $\left(\varphi \sim 0^{\circ}\right)$ exhibiting the expected set of reflections at $\psi \sim 60^{\circ}$ from the symmetric group of planes.

Fig. 3 a), b) and c) shows the morphology of the polished surface in the longitudinal direction of the fibers grown by LFZ at 25, 50 and

Table 1

Structural parameters obtained from Rietveld analysis of LFZ fiber grown at 25, 50 and 100 $\mathrm{mm} / \mathrm{h}$.

\begin{tabular}{|c|c|c|c|c|c|c|c|}
\hline $\begin{array}{l}\text { Growth } \\
\text { speed }\end{array}$ & $\begin{array}{l}\% \text { vol. } \\
\mathrm{BiFeO}_{3}\end{array}$ & $a=b$ & $c$ & $\begin{array}{l}\text { \% vol. } \\
\mathrm{Bi}_{25} \mathrm{FeO}_{40}\end{array}$ & $\boldsymbol{a}$ & $\mathrm{R}_{\mathrm{p}}$ & $\mathrm{R}_{\mathrm{wp}}$ \\
\hline$(\mathrm{mm} / \mathrm{h})$ & $R 3 c h$ & $(\mathrm{~nm})$ & $(\mathrm{nm})$ & $I 23$ & $(\mathrm{~nm})$ & & \\
\hline 25 & 63 & 0.5576 & 1.387 & 37 & 1.0176 & 10.6 & 15.5 \\
\hline 50 & 62 & 0.5578 & 1.387 & 38 & 1.0176 & 8.8 & 11.5 \\
\hline 100 & 58 & 0.5579 & 1.388 & 42 & 1.0176 & 9.7 & 12.6 \\
\hline
\end{tabular}


a)

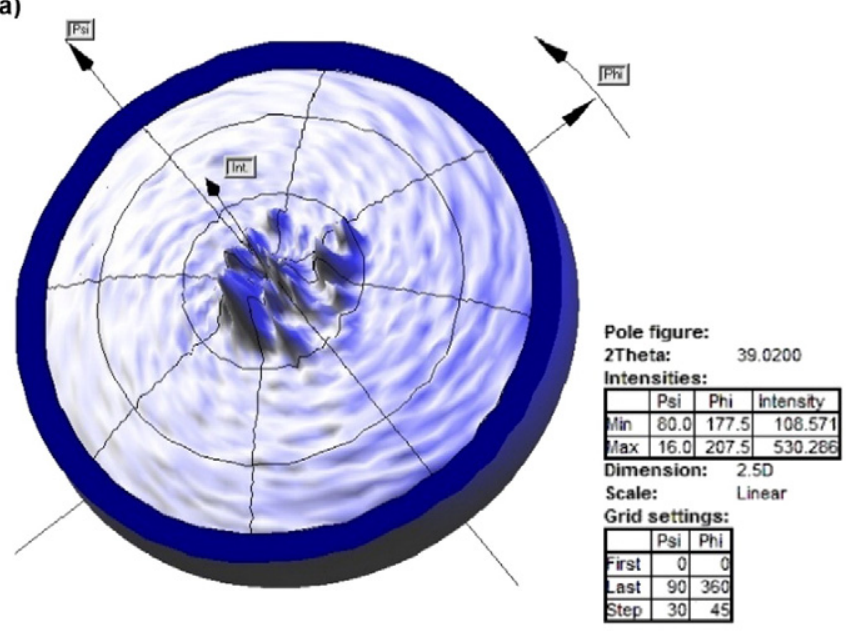

b)

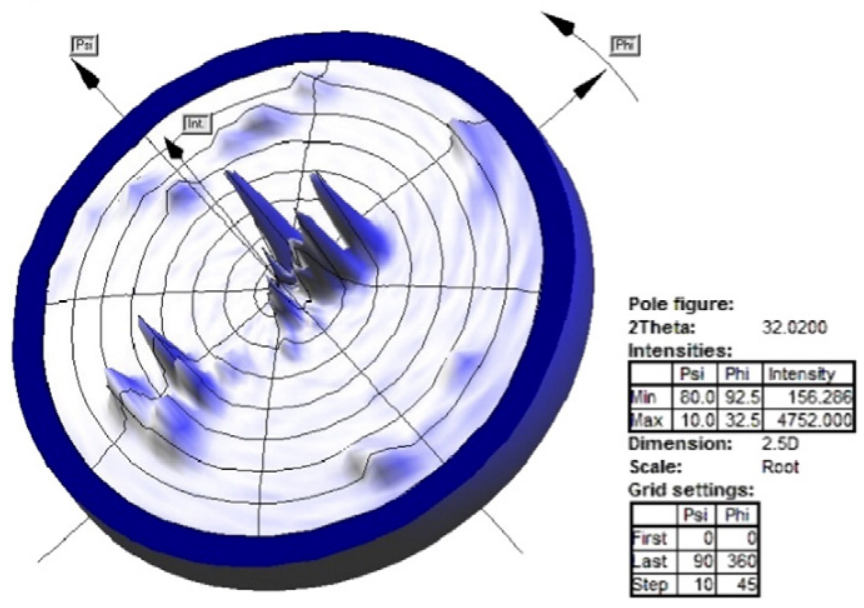

Fig. 2. Pole figures of fiber grown at $25 \mathrm{~mm} / \mathrm{h}$; a) at $2 \boldsymbol{\theta}=39.02^{\circ}$ of $\left(\begin{array}{ll}0 & 0\end{array}\right) \mathrm{BiFeO}_{3}$ planes from a transversal section; b) at $2 \boldsymbol{\theta}=32.02^{\circ}$ of $\left(\begin{array}{lll}1 & 1 & 0\end{array}\right) \mathrm{BiFeO}_{3}$ planes from a longitudinal section.

$100 \mathrm{~mm} / \mathrm{h}$ respectively. As confirmed by local EDS, the darker areas correspond to the perovskite bismuth ferrite phase ( $|\mathrm{Bi}| /|\mathrm{Fe}| \sim 1)$, separated by the brighter grainy regions, found to be the denser bismuth enriched secondary phase $(|\mathrm{Bi}| /|\mathrm{Fe}| \sim 25)$. Nonetheless a quantitative analysis in terms of overall surface ratios would not necessarily translate the volume distribution of phases in the fiber, due to the anisotropic and dendritic arrangement of both crystallites. Besides the growth of two mentioned major phases, for higher growth rates (150 and $200 \mathrm{~mm} / \mathrm{h}$ not shown) it can be detected formations of scarce and scattered small iron rich regions (darkest grains) that marginal appear on the fiber grown at $100 \mathrm{~mm} / \mathrm{h}$. In the sequence of images in Fig. 3, the rather chaotic dispersion of crystallites sizes, shapes and arrangements observed for the fiber grown at $100 \mathrm{~mm} / \mathrm{h}$ progresses through the fiber grown at $50 \mathrm{~mm} / \mathrm{h}$ to a relative alignment and patterning of the constituent crystallites along the longitudinal direction of the fiber found on the fiber grown at $25 \mathrm{~mm} / \mathrm{h}$. Qualitatively, the crystallites formed in the sample grown at $25 \mathrm{~mm} / \mathrm{h}$ appear relatively more prolonged and aligned when compared to those grown with $50 \mathrm{~mm} / \mathrm{h}$ and $100 \mathrm{~mm} / \mathrm{h}$.

Fig. 4 shows the susceptibility vs. temperature curves for the fiber grown by LFZ at $25 \mathrm{~mm} / \mathrm{h}$ rate, in field cooling (FC) and zero field cooling (ZFC) modes obtained at a constant magnetic field of 0.5 Tesla. The measurements were performed in two distinct geometries, in a perpendicular $(\perp)$ and in a parallel $(/ /)$ positioning of fiber growth axis $(\boldsymbol{z})$ in relation to the external magnetic field $(\boldsymbol{B})$. The overall susceptibility increases when the fiber is parallel to the external magnetic field, a)

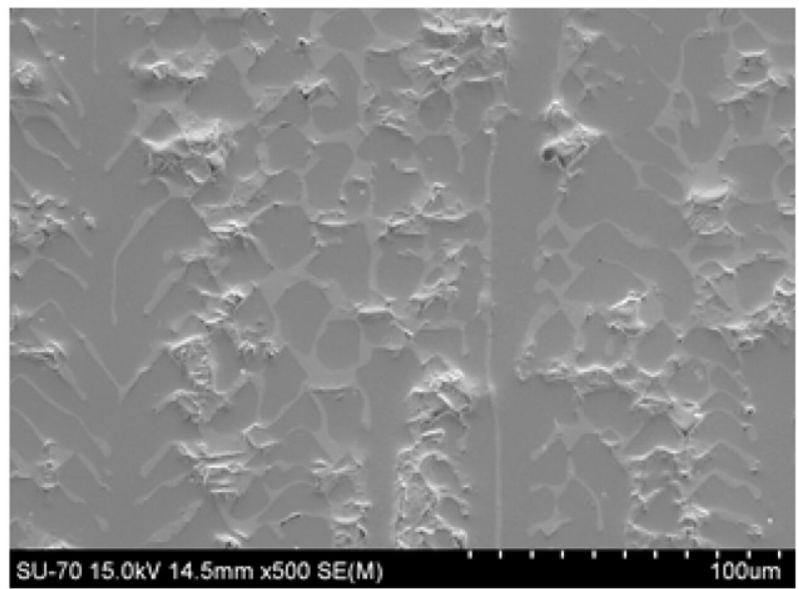

b)

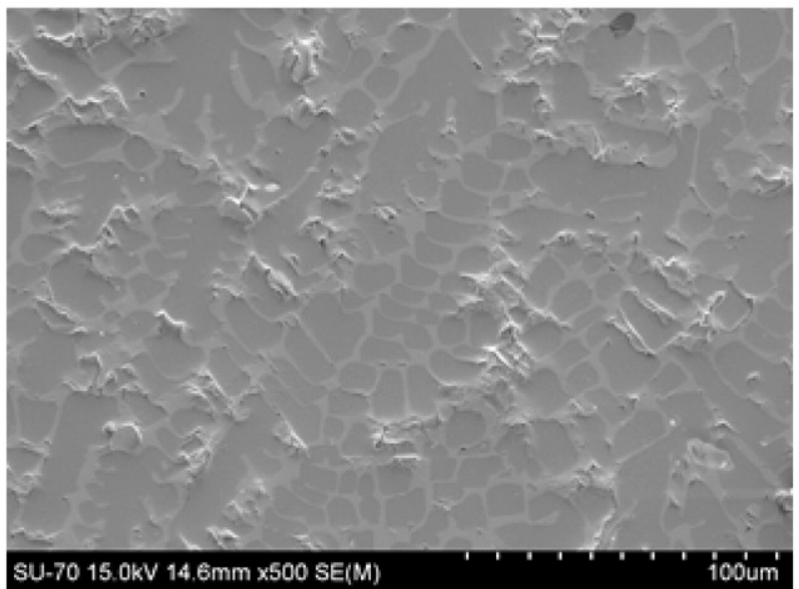

c)

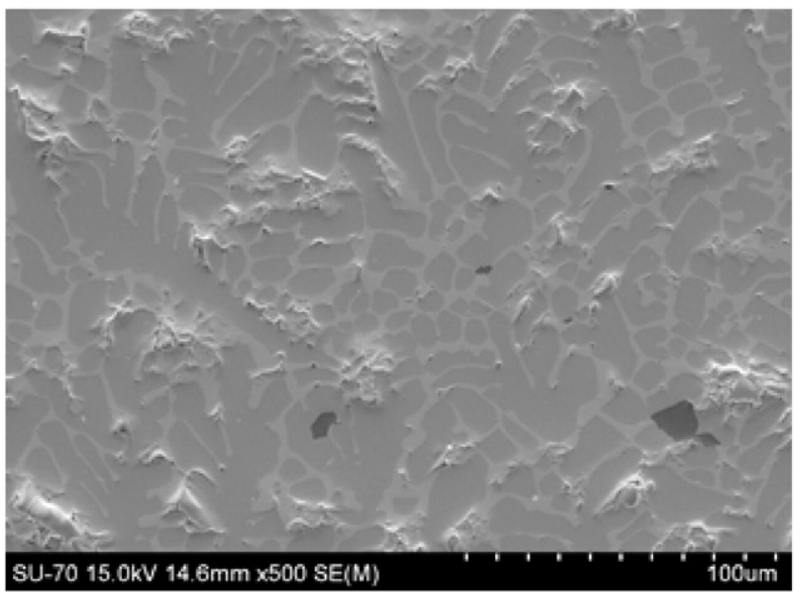

Fig. 3. Comparison of SEM images obtained in the longitudinal direction of fibers grown at a) 25 , b) 50 and c) $100 \mathrm{~mm} / \mathrm{h}$.

while a comparison of difference between the FC and ZFC susceptibilities almost disappears in this geometry; however, it becomes substantial for the perpendicular configuration. Analyzing in detail the behavior of the susceptibility of FC and ZFC curves performed in the fibers parallel to the magnetic field, it is possible to relate a conventional Curie-Weiss model " $\boldsymbol{\chi}=\mathbf{C} /(\mathbf{T}-\boldsymbol{\Theta})$ " from which the Weiss constant $(\boldsymbol{\theta})$ $\sim 283 \mathrm{~K}$ and the Curie constant $(\mathbf{C}) \sim 52.7 \mathrm{~K}^{-1}$ can be estimated, indicating a noteworthy magnetic exchange interaction. 
a)

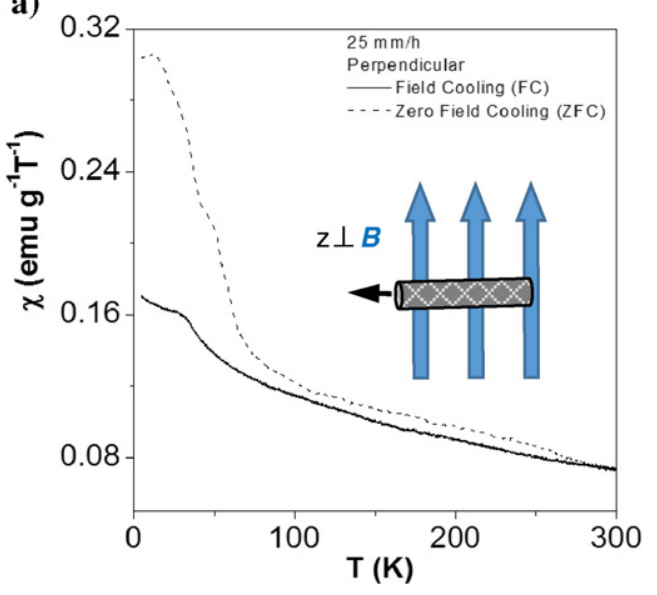

b)

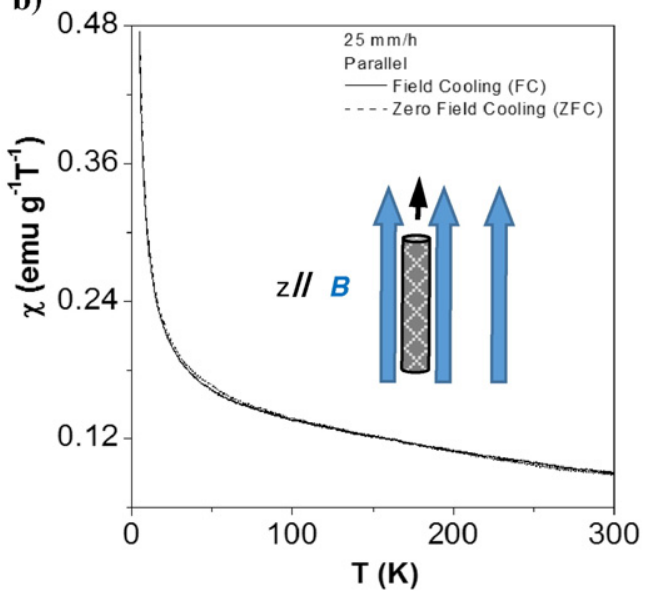

Fig. 4. Temperature variation of susceptibility for field cooling (FC) and zero field cooling (ZFC) measurement of fiber grown at $25 \mathrm{~mm} / \mathrm{h}$ placed in (a) perpendicular $(\perp)$ and (b) parallel (//) to the external magnetic field.

Considering that, the mechanism that masks the FC and ZFC curves when the samples are parallel to the applied magnetic field is the same that boosts a detachment for the perpendicular configuration. Some hypothesis can be discussed and analyzed: These sets of samples run in a relative low susceptibility regime, as such, the shape factor of the fiber turns to be negligible. Nonetheless, the samples were cut in controlled height to diameter ratio in order to prevent misreading's. The difference between the FC and ZFC susceptibilities increases when the fiber is perpendicular to the external field. At first, this resembles canonical spin glass behavior [33] since the peak temperature of ZFC magnetization is equal to the bifurcation temperature between the FC and ZFC curves.

However, these features are not observed when the fiber is kept parallel to the external field, neither any considerable difference or bifurcation was visible for the samples grown at higher rates. These observations are compatible with a contribution to the magnetic anisotropy resulting from the preferable alignment of BFO magnetization easy axis along the fibber, in contrast with an isotropic response expected from disordered crystallites.

Fig. 5 shows the variation of magnetic moment with field, at $5 \mathrm{~K}$ and $300 \mathrm{~K}$ with the external magnetic field perpendicular to the sample axis, it is visible from the curves that the common behavior of the samples at $300 \mathrm{~K}$ is paramagnetic. At $5 \mathrm{~K}$, the behavior become more complex, a combination of paramagnetism positive slope, with sa ferrimagnetism contribution suggested by a straitened loop at low fields (inset in Fig. $5 \mathrm{~b})$, besides the expected antiferromagnetism from the stoichiometric $\mathrm{BiFeO}_{3}$ phase. a)

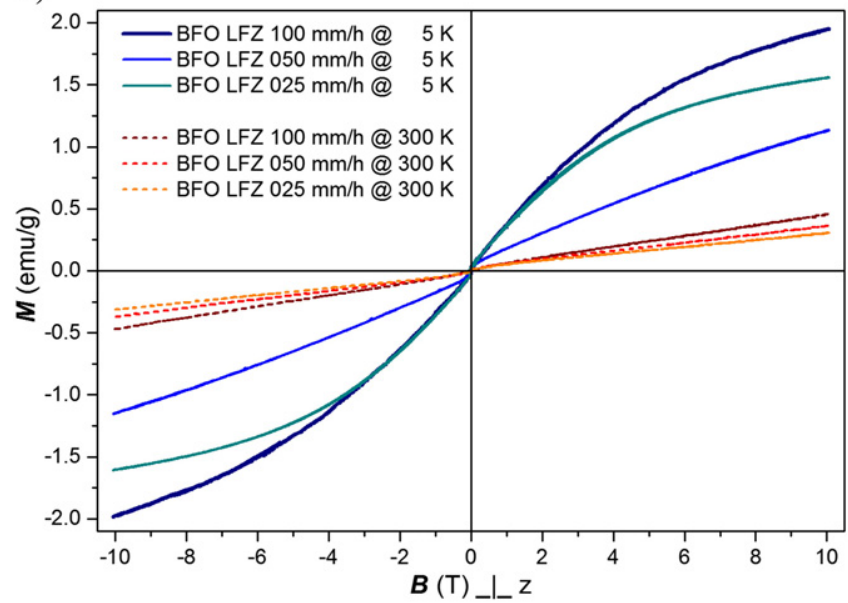

b)

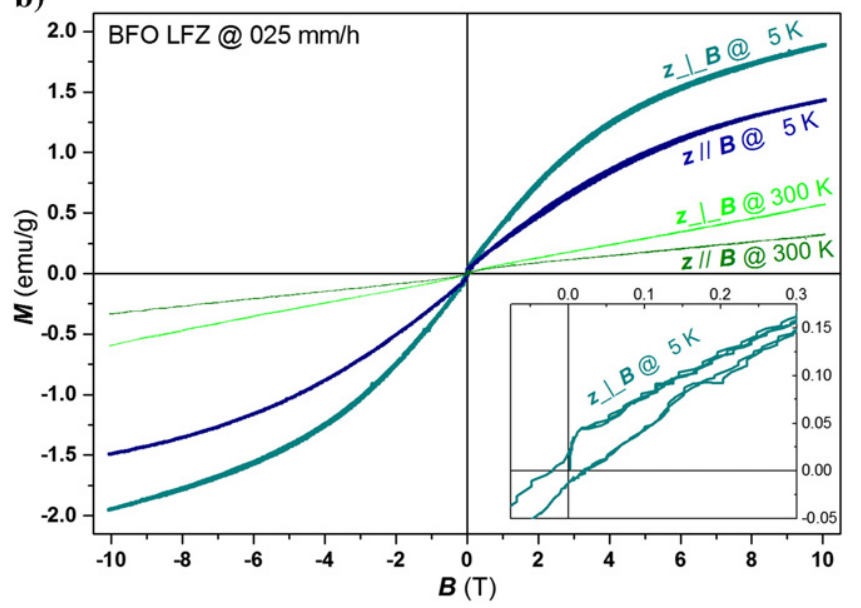

Fig. 5. Susceptibility vs. magnetic field at 5 and $300 \mathrm{~K}$, a) comparison between fibers grown at 25,50 and $100 \mathrm{~mm} / \mathrm{h}$ in a perpendicular $(\perp)$ geometry. b) Comparison of measurements in perpendicular $(\perp)$ and parallel $(/ /)$ geometries for the fiber grown at the rate $25 \mathrm{~mm} / \mathrm{h}$. Inset: small hysteresis detail.

The most consistent explanation, of the anomalies observed in the magnetization signals exceeding the conventional small response of the antiferromagnetic $\mathrm{BiFeO}_{3}$ matrix and the diamagnetism of the bismuth rich phase compound, are usually driven by irregular magnetic ions within the materials; which can be contaminants or intrinsic elements under local stoichiometry deviations. These are usually near defects, grain boundaries or oxygen deficiencies, also can prompt at magnetic inhomogeneities induced by domain walls or crystalorientation-mismatches, among other structural distortions [34]. In this context and as consequence of the LFZ technique, the extents of Fe ions that can be available to participate in a paramagnetic contribution are mostly disperse in the fraction of $\mathrm{Bi}_{25} \mathrm{FeO}_{40}$ phase, whereas a ferrimagnetic component can have origin by the collective response of non-compensated spins of neighboring $\mathrm{Fe}$ ions from the $\mathrm{BiFeO}_{3}$ phase. These asymmetric magnetic environments can manifest mainly along the interfaces between the two different phases, which are in fact, very abundant in the present samples, as sustained by XRD analysis and attested by SEM images. Therefore, the gross amount of "more susceptible" Fe ions is directly proportional to the total area of interfaces between the two different phases, in its turn, has a complex dependency on the distribution of grains sizes and shapes factors and type of percolation between the constituent phases. Moreover, the relative orientation between crystallites and their collective alignment along the fibers axis play a relevant role in the magnetic susceptibility anisotropy of the samples. 
It is manifest that the main contribution to the magnetic response of this system ought to be related to the interfaces between the two segregated phases of Bismuth ferrite and the diamagnetic bismuth enriched phase. At these interfaces occurs a pertinent structural asymmetry, imposing a break of the $\mathrm{BiFeO}_{3}$ antiferromagnetic matrix, hence settling a distribution of unbalanced spins along these surfaces. Hereafter, the overall susceptibility can be enhanced by favoring the extent of interfaces between the two distinct phases; this can be achieved by reducing grains growth by slightly increasing the pulling rate, while still ensuring adequate phases segregation. An anisotropic response can originate in the position of the interfaces, function of grains size, shape and relative alignment. Moreover, the increase in magnetic anisotropy of fibers is a direct consequence of reducing the growth speed, allowing the necessary time for the crystallites to be driven by the heat flux during the solidification and the temperature gradient from the highly heated molten zone [35]. As result, the common orientation of grain boundaries between phases is favored; in addition, the $\mathrm{BiFeO}_{3}$ hexagonal phase forms with a $\boldsymbol{c}$ axis preferred orientation along the longitudinal direction ensuing a narrower scattering of $\boldsymbol{a}$ and $\boldsymbol{b}$ magnetization easy axis [7] perpendicular to the fiber; hence explaining the higher susceptibility of a perpendicular geometry to the magnetic field.

\section{Conclusions}

In this work, we have grown fibers of Bismuth Ferrite by using the laser floating zone technique varying the growth rate. Decreasing the pulling rate of the fibers has a minor effect on the overall composition of fibers, while the Bismuth enriched secondary phase namely, $\mathrm{Bi}_{25} \mathrm{FeO}_{40}$ is more accounted in the fibers grown at the highest speed. The fibers exhibit a paramagnetic behavior in the high temperature regions with a combination of mixed ferrimagnetic and antiferromagnetic contribution in the low temperature region. The magnetic susceptibility is strongly influenced by the relative orientation of the fiber axis to the external magnetic field, due to the modulated microstructure. The relative orientations of the crystalline $\boldsymbol{c}$ axis and phase's grain boundaries are mainly distributed along the longitudinal axis of the fibers, enhanced by LFZ processing at slower growth speed.

\section{Acknowledgments}

This work was developed in the scope of the project I3N (UID/CTM/ 50025/2013), CICECO-Aveiro Institute of Materials (UID/CTM/50011/ 2013), grants SFRH/BPD/75588/2010 and SFRH/BPD/80663/2011 financed by national funds through the FCT/MEC and when applicable co-financed by FEDER under the PT2020 Partnership Agreement.

\section{References}

[1] C.N.R. Rao, B. Raveau, Transition Metal Oxides, 2nd Ed. Wiley VCH, Weinheim, 1995 1998.

[2] D. Norton, Mater. Sci. Eng. R 43 (2004) 139-247.

[3] W. Eerenstein, N.D. Mathur, J.F. Scott, Nature 442 (2006) 759-765.

4] M. Fiebig, J. Phys. D 38 (2005) R123-R152.

[5] H. Schmid, Ferroelectrics 162 (1994) 317-338.

[6] F. Zavaliche, S.Y. Yang, T. Zhao, Y.H. Chu, M.P. Cruz, C.B. Eom, et al., Phase Transit. 79 (2006) 991-1017.

[7] I. Sosnowska, T.P. Neumaier, E. Steichele, J. Phys. C 15 (1982) 4835-4846.

[8] I.E. Dzyaloshinskii, Sov. Phys. - JETP 5 (1957) 1259-1272.

[9] T. Moriya, Phys. Rev. 120 (1960) 91-98.

[10] J. Wang, J.B. Neaton, H. Zheng, V. Nagarajan, S.B. Ogale, B. Liu, et al., Science 299 (2003) 1719-1722.

[11] Q. He, C.H. Yeh, J.C. Yang, G.S. Bhalla, C.W. Liang, P.W. Chiu, et al., Phys. Rev. Lett. 108 (2012) 067203.

[12] L.W. Martin, Y.H. Chu, M.B. Holcomb, M. Huijben, S.J. Han, D. Lee, et al., Nano Lett. 8 (2008) 2050-2055.

[13] M. Daraktchiev, G. Catalan, J.F. Scott, Ferroelectrics 375 (2008) 22-31.

[14] G. Catalan, J.F. Scott, Adv. Mater. 21 (2009) 2463-2485.

[15] S. Ghosh, S. Dasgupta, A. Sen, H.S. Maiti, Mater. Res. Bull. 40 (2005) 2073-2079.

[16] A.K. Pradhan, et al., J. Appl. Phys. 97 (2005) 093903.

[17] D. Thapa, V.R. Palkar, M.B. Kurup, S.K. Malik, Mater. Lett. 58 (2004) 2692-2694.

[18] Y.P. Wang, L. Zhou, M.F. Zhang, X.Y. Chen, J.M. Liu, Z.G. Liu, Appl. Phys. Lett. 84 (2004) 1731-1733.

[19] S. Iakovlev, C.-H. Solterbeck, M. Kuhnke, M. Es-Souni, J. Appl. Phys. 97 (2005) 094901.

[20] I.A. Santos, H.L.C. Grande, V.F. Freitas, S.N. de Medeiros, A. Paesano Jr., L.F. Cótica, E. Radovanovic, J. Non-Cryst. Solids 352 (2006) 3721-3724.

[21] M.R.N. Soares, C. Nico, M. Peres, N. Ferreira, A.J.S. Fernandes, T. Monteiro, F.M. Costa, J. Appl. Phys. 109 (2011) 013516.

[22] R.G. Carvalho, A.J.S. Fernandes, F.J. Oliveira, E. Alves, N. Franco, C. Louro, R.F. Silva, F.M. Costa, J. Eur. Ceram. Soc. 30 (2010) 3311-3318.

[23] N.M. Ferreira, S. Rasekh, F.M. Costa, M.A. Madre, A. Sotelo, J.C. Diez, M.A. Torres, Mater. Lett. 83 (2012) 144-147.

[24] F. Amaral, L.C. Costa, M.A. Valente, A.J.S. Fernandes, N. Franco, E. Alves, F.M. Costa, Acta Mater. 59 (2011) 102-111.

[25] H. Eisaki, N. Kaneko, D.L. Feng, A. Damascelli, P.K. Mang, K.M. Shen, Z.-X. Shen, M. Greven, Phys. Rev. B 69 (2004) 064512.

[26] M.F. Carrasco, V.S. Amaral, R.F. Silva, F.M. Costa, Appl. Surf. Sci. 257 (12) (2011) 5283-5286 (1)

[27] E. Barzi, L. del Frate, D. Turrioni, R. Johnson, M. Kuchnir, Adv. Cryog. Eng. 824 (2006) 416-424.

[28] S.K. Pradhan, B.K. Roul, Phys. B Condens. Matter 406 (2011) 3313-3317.

[29] N.M. Ferreira, A.V. Kovalevsky, J.C. Waerenborgh, M. Quevedo-Reyes, A.A. Timopheev, F.M. Costa, J.R. Frade, J. Alloys Compd. 611 (2014) 57-64.

[30] T.T. Carvalho, J.R.A. Fernandes, J.P. de la Cruz, J.V. Vidal, N.A. Sobolev, F. Figueiras, S. Das, V.S. Amaral, A. Almeida, J.A. Moreira, P.B. Tavares, J. Alloys Compd. 554 (2013) 97-103.

[31] Y.N. Venevtsev, V.V. Gagulin, Inorg. Mater. 31 (1995) 797-810.

[32] V.V. Volkov, V.Y. Endrzheevskaya, Y.F. Kargin, V.M. Skorikov, Russ. J. Inorg. Chem. 34 (1989) 1787-1789.

[33] V. Bisht, K.P. Rajeev, S. Banerjee, Solid State Commun. 150 (2010) 884-887.

[34] R. Mahesh, R. Mahendiran, A.K. Raychaudhuri, C.N.R. Rao, Appl. Phys. Lett. 68 (1996) 2291-2293.

[35] J.M. Vieira, R.A. Silva, R.F. Silva, F.M. Costa, Appl. Surf. Sci. 258 (2012) 9175-9180. 